\title{
LONG-TERM MONITORING OF MRK 501 FOR ITS VERY HIGH ENERGY $\gamma$ EMISSION AND A FLARE IN 2011 OCTOBER
}

\author{
B. Bartoli ${ }^{1,2}$, P. Bernardini ${ }^{3,4}$, X. J. Bi ${ }^{5}$, C. Bleve ${ }^{3,4}$, I. Bolognino ${ }^{6,7}$, P. Branchini ${ }^{8}$, A. Budano ${ }^{8}$, \\ A. K. Calabrese Melcarne ${ }^{9}$, P. Camarri ${ }^{10,11}$, Z. CaO ${ }^{5}$, R. Cardarelli ${ }^{11}$, S. Catalanotti ${ }^{1,2}$, C. Cattaneo ${ }^{7}$, S. Z. Chen $^{5}$, \\ T. L. Chen ${ }^{12}$, Y. Chen ${ }^{5}$, P. Creti ${ }^{4}$, S. W. Cui ${ }^{13}$, B. Z. Dai ${ }^{14}$, G. D’Alí Staiti ${ }^{15,16}$, Danzengluobu ${ }^{12}$, M. Dattoli ${ }^{17,18,19}$, \\ I. De Mitri ${ }^{3,4}$, B. D’Ettorre Piazzoli ${ }^{1,2}$, T. Di Girolamo ${ }^{1,2}$, X. H. Ding ${ }^{12}$, G. Di Sciascio ${ }^{11}$, C. F. FenG $^{20}$, ZhaOYang FenG $^{5}$,

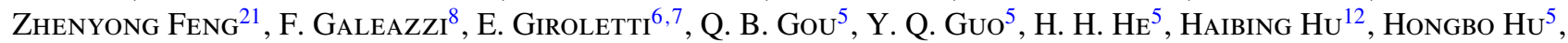

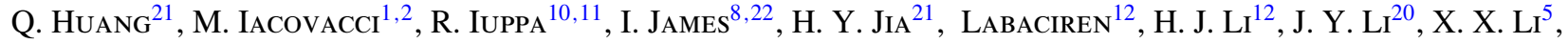

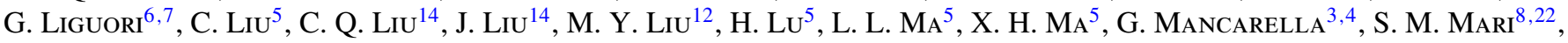 \\ G. Marsella ${ }^{3,4}$, D. Martello ${ }^{3,4}$, S. Mastroianni ${ }^{2}$, P. Montini ${ }^{8,22}$, C. C. Ning ${ }^{12}$, A. Pagliaro ${ }^{16,23}$, M. Panareo ${ }^{3,4}$,

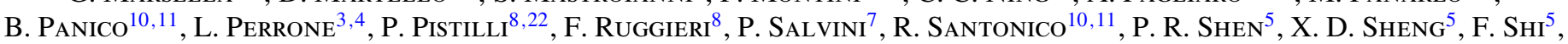

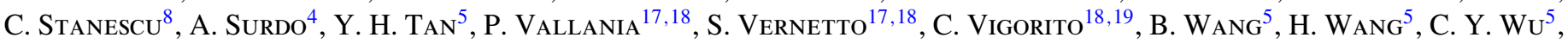

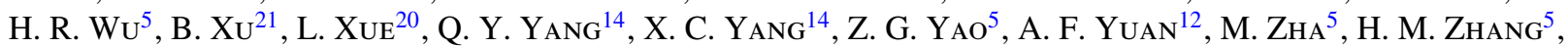

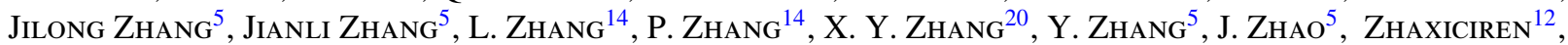 \\ ZhaXisAngZhU ${ }^{12}$, X. X. ZhOU ${ }^{21}$, F. R. ZHU ${ }^{21}$, Q. Q. ZHU ${ }^{5}$, AND G. ZIZZI ${ }^{9}$ \\ (The ARGO-YBJ Collaboration) \\ ${ }^{1}$ Dipartimento di Fisica dell'Università di Napoli “Federico II," Complesso Universitario di Monte Sant'Angelo, via Cinthia, I-80126 Napoli, Italy \\ ${ }^{2}$ Istituto Nazionale di Fisica Nucleare, Sezione di Napoli, Complesso Universitario di Monte Sant'Angelo, via Cinthia, I-80126 Napoli, Italy \\ ${ }^{3}$ Dipartimento di Matematica e Fisica “E. De Giorgi” dell'Università del Salento, via per Arnesano, I-73100 Lecce, Italy \\ ${ }^{4}$ Istituto Nazionale di Fisica Nucleare, Sezione di Lecce, via per Arnesano, I-73100 Lecce, Italy \\ ${ }^{5}$ Key Laboratory of Particle Astrophysics, Institute of High Energy Physics, Chinese Academy of Sciences, P.O. Box 918, 100049 Beijing, China; chensz@ihep.ac.cn \\ ${ }^{6}$ Dipartimento di Fisica Nucleare e Teorica dell’Università di Pavia, via Bassi 6, I-27100 Pavia, Italy \\ ${ }^{7}$ Istituto Nazionale di Fisica Nucleare, Sezione di Pavia, via Bassi 6, I-27100 Pavia, Italy \\ ${ }^{8}$ Istituto Nazionale di Fisica Nucleare, Sezione di Roma Tre, via della Vasca Navale 84, I-00146 Roma, Italy \\ ${ }^{9}$ Istituto Nazionale di Fisica Nucleare-CNAF, Viale Berti-Pichat 6/2, I-40127 Bologna, Italy \\ ${ }^{10}$ Dipartimento di Fisica dell’Università di Roma "Tor Vergata," via della Ricerca Scientifica 1, I-00133 Roma, Italy \\ ${ }^{11}$ Istituto Nazionale di Fisica Nucleare, Sezione di Roma Tor Vergata, via della Ricerca Scientifica 1, I-00133 Roma, Italy \\ 12 Tibet University, 850000 Lhasa, Xizang, China \\ ${ }^{13}$ Hebei Normal University, Shijiazhuang 050016, Hebei, China \\ ${ }^{14}$ Yunnan University, 2 North Cuihu Rd., 650091 Kunming, Yunnan, China \\ ${ }^{15}$ Dipartimento di Fisica e Tecnologie Relative, Università degli Studi di Palermo, Viale delle Scienze, Edificio 18, I-90128 Palermo, Italy \\ ${ }^{16}$ Istituto Nazionale di Fisica Nucleare, Sezione di Catania, Viale A. Doria 6, I-95125 Catania, Italy \\ ${ }^{17}$ Osservatorio Astrofisico di Torino, Istituto Nazionale di Astrofisica, corso Fiume 4, I-10133 Torino, Italy \\ ${ }^{18}$ Istituto Nazionale di Fisica Nucleare, Sezione di Torino, via P. Giuria 1, I-10125 Torino, Italy \\ ${ }^{19}$ Dipartimento di Fisica Generale dell'Università di Torino, via P. Giuria 1, I-10125 Torino, Italy \\ ${ }^{20}$ Shandong University, 250100 Jinan, Shandong, China \\ ${ }^{21}$ Southwest Jiaotong University, 610031 Chengdu, Sichuan, China \\ ${ }^{22}$ Dipartimento di Fisica dell'Università "Roma Tre," via della Vasca Navale 84, I-00146 Roma, Italy \\ ${ }^{23}$ Istituto di Astrofisica Spaziale e Fisica Cosmica dell' Istituto Nazionale di Astrofisica, via La Malfa 153, I-90146 Palermo, Italy \\ Received 2012 March 8; accepted 2012 July 27; published 2012 September 18
}

\begin{abstract}
As one of the brightest active blazars in both X-ray and very high energy $\gamma$-ray bands, Mrk 501, is very useful for physics associated with jets from active galactic nuclei. The ARGO-YBJ experiment has monitored Mrk 501 for $\gamma$ rays above $0.3 \mathrm{TeV}$ since 2007 November. The largest flare since 2005 was observed from 2011 October and lasted until about 2012 April. In this paper, a detailed analysis of this event is reported. During the brightest $\gamma$-ray flaring episodes from 2011 October 17 to November 22, an excess of the event rate over $6 \sigma$ is detected by ARGO-YBJ in the direction of Mrk 501, corresponding to an increase of the $\gamma$-ray flux above $1 \mathrm{TeV}$ by a factor of $6.6 \pm 2.2$ from its steady emission. In particular, the $\gamma$-ray flux above $8 \mathrm{TeV}$ is detected with a significance better than $4 \sigma$. Based on time-dependent synchrotron self-Compton (SSC) processes, the broadband energy spectrum is interpreted as the emission from an electron energy distribution parameterized with a single power-law function with an exponential cutoff at its high-energy end. The average spectral energy distribution for the steady emission is well described by this simple one-zone SSC model. However, the detection of $\gamma$-rays above $8 \mathrm{TeV}$ during the flare challenges this model due to the hardness of the spectra. Correlations between X-rays and $\gamma$-rays are also investigated.
\end{abstract}

Key words: BL Lacertae objects: individual (Markarian 501) - galaxies: active - gamma rays: general - radiation mechanisms: non-thermal

Online-only material: color figures

\section{INTRODUCTION}

Blazars, including BL Lac objects and flat-spectrum radio quasars, are the most extreme subclass of active galactic nuclei
(AGNs). Most of the identified extragalactic $\gamma$-ray sources belong to this category. Their emission is believed to be dominated by non-thermal and strongly Doppler-boosted radiation from a relativistic jet of magnetized plasma which is aligned along our 
line of sight. The physical mechanism for the production of their $\gamma$-ray emission is still under debate. Leptonic models attribute the $\gamma$-ray emission to inverse Compton scattering of the synchrotron (synchrotron self-Compton, SSC) or external photons (external Compton) by the same population of relativistic electrons (Ghisellini et al. 1998; Dermer et al. 1992; Sikora et al. 1994), therefore an X-ray/ $\gamma$-ray correlation is expected. The lack of strong emission lines in the radiation from BL Lac objects is taken as evidence of ambient photons playing a minor role (e.g., Krawczynski 2004), and hence the SSC model is favored. Hadronic models attribute the $\gamma$-ray emission to proton-initiated cascades and/or proton-synchrotron emission in a magnetic field-dominated jet (Aharonian 2000). However, the tight X-ray and very high energy (VHE) $\gamma$-ray correlation and the very rapid $\gamma$-ray variability are taken as strong challenges to models based on hadronic processes. Recently, a longterm continuous monitoring of Mrk 421 has been performed based on the ARGO-YBJ experiment and satellite-borne X-ray detectors (Bartoli et al. 2011a). According to this investigation, both the temporal and the spectral results generally favor the SSC model. Even in the framework of the SSC model, the fundamental question referred to the origin of the flux and spectral variability, observed on timescales from minutes to tens of years, is still open to discussion.

Mrk $501(z=0.034)$ was discovered with VHE emission by the Whipple collaboration (Quinn et al. 1996). It is one of the best-studied blazars with extensive studies on various timescales. In 1997, Mrk 501 went into a state with surprisingly high activity and strong variability and became more than a factor of 10 brighter (above $1 \mathrm{TeV}$ ) than the Crab Nebula (Djannati-Atai et al. 1999; Aharonian et al. 1999; Amenomori et al. 2000). The fastest $\gamma$-ray flux variability on a timescale of minutes was observed in 2005 (Albert et al. 2007). Significant spectral variability was detected with the harder spectrum at brighter states compared to low-activity states (Albert et al. 2007; Anderhub et al. 2009; Acciari et al. 2011). In the middle of 2009, a multi-frequency observational campaign of Mrk 501 was carried out for 4.5 months with excellent energy coverage from radio to VHE $\gamma$-ray when it underwent low activity (Abdo et al. 2011). Throughout the campaign, the source was sampled quite uniformly in all wavelength bands except for the VHE band because the Cherenkov Telescopes cannot operate during non-optimal weather conditions or periods of bright moonlight. In 2011 October, Mrk 501 underwent a strong flare detected by the MAXI satellite in X-rays (Sootome et al. 2011) and by the ARGO-YBJ detector in VHE $\gamma$-rays (Bartoli et al. 2011c).

To understand the variability of emission and the underlying acceleration and radiation mechanisms in jets, continuous multiwavelength observations from the X-ray to the VHE $\gamma$-ray band are crucial, especially over a very long term. The broadband energy spectra could provide constraints on the parameters of the models. The Cherenkov Telescopes cannot constantly monitor AGNs because of their limited duty cycle and narrow field of view (FOV). The wide-FOV ARGO-YBJ detector, operated with a high duty cycle ( $>85 \%)$, is more suitable for monitoring. Working at energies above $300 \mathrm{GeV}$, ARGO-YBJ extends the multi-wavelength survey carried out by the satellite-borne $\mathrm{X}$-ray detector Swift and the GeV $\gamma$-ray detector Fermi-LAT. Particularly, the spectral energy distribution (SED) of Mrk 501 is covered without any gap from $100 \mathrm{MeV}$ to $10 \mathrm{TeV}$. All the measurements would set strong constraints on the model of AGN emission.
In this paper, we report on the multi-wavelength view of the emission from Mrk 501 from 2008 August to 2012 April, including the average spectra during quasi-steady and flaring periods.

\section{THE ARGO-YBJ EXPERIMENT AND DATA ANALYSIS}

The ARGO-YBJ experiment, located at the Cosmic Ray Laboratory of Yangbajing (Tibet, China) at an altitude of $4300 \mathrm{~m}$ a.s.l., is the result of a collaboration among Chinese and Italian institutions and is designed for VHE $\gamma$-ray astronomy and cosmic-ray observations. The detector consists of a single layer of resistive plate chambers (RPCs), which are equipped with charge readout strips $(6.75 \mathrm{~cm} \times 61.80 \mathrm{~cm}$ each $)$. The logical OR of signals from eight neighboring strips constitutes the pixel (called a "pad") for triggering and timing purposes. Each RPC is read with 10 pixels. One hundred thirty clusters (each composed of 12 RPCs) are installed to form a carpet of about $5600 \mathrm{~m}^{2}$ with an active area of $\sim 93 \%$. This central carpet is surrounded by 23 additional clusters (the "guard ring"). The total area of the array is $110 \mathrm{~m} \times 100 \mathrm{~m}$. The ARGO-YBJ detector is operated by requiring the number of fired pads $\left(N_{\text {pad }}\right)$ to be at least 20 within $420 \mathrm{~ns}$ on the entire carpet. The trigger rate is $3.5 \mathrm{kHz}$ with a dead time of $4 \%$. The angular resolution, pointing accuracy, and stability of the ARGO-YBJ detector array have been thoroughly tested by measuring the shadow of the Moon in cosmic rays (Bartoli et al. 2011b). The absolute energy scale uncertainty is less than $13 \%$ for all measured cosmic-ray showers (Bartoli et al. 2011b). More details about the detector and the RPC performance can be found in Aielli et al. (2006, 2009a, 2009b, 2009c).

For the multi-wavelength investigation together with FermiLAT, the data collected by ARGO-YBJ after 2008 August when Fermi was launched are used. The total effective observation time is 1179.6 days. To achieve a good angular resolution, events with zenith angles less than $50^{\circ}$ are used, and further selection criteria (Bartoli et al. 2011a, 2012) are applied. The total number of events after filtering is $1.86 \times 10^{11}$ for this work. No $\gamma$ /hadron discrimination is applied. The opening angle $\psi_{70}$, which contains $71.5 \%$ of the events from a point-like source, is 1.36 for events with $N_{\text {pad }}>60$. In order to remove the effect of the cosmic-ray anisotropy, the method described in Bartoli et al. (2011a, 2012) was applied. The significance of the excess is estimated using the $\mathrm{Li} \& \mathrm{Ma}$ (1983) method. With this data analysis, the significance of the excess observed from the direction of the Crab Nebula is 17 standard deviations $(\sigma)$ in 3.5 years, which indicates that the 3.5 year cumulative $5 \sigma$ sensitivity of ARGO-YBJ has reached $0.3 \mathrm{Crab}$ for point sources (Cao \& Chen 2011).

\section{RESULTS}

\subsection{Light Curves}

The daily flux from Mrk 501 at energy $15-50 \mathrm{keV}$ provided by Swift $/$ BAT $^{24}$ is publicly available and used in this work. The light curve from 2008 August to 2012 April is shown in panel (a) of Figure 1 with a bin size of 30 days. The best fit with a constant value for the light curve is $(8.9 \pm 0.4) \times 10^{-4}$ counts $\mathrm{cm}^{-2} \mathrm{~s}^{-1}$ with a $\chi^{2}$ of 492.9 for 44 degrees of freedom (ndf). A significant feature is the flare at the end of 2011 with the flux enhanced by a factor of about four. Without data during the flaring

\footnotetext{
${ }^{24}$ Transient monitor results provided by the Swift/BAT team: http://heasarc.gsfc.nasa.gov/docs/swift/results/transients/weak/Mrk501/.
} 


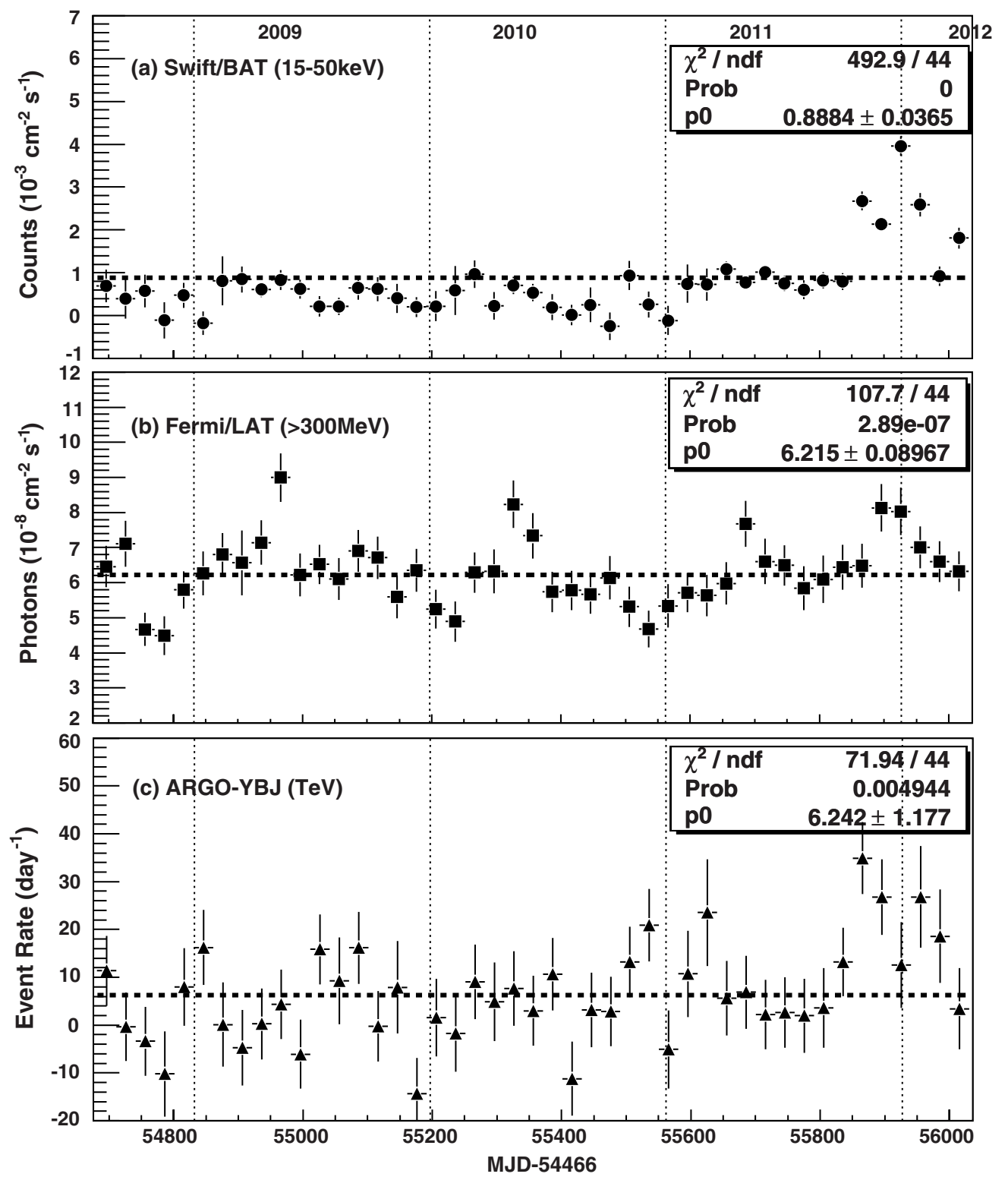

Figure 1. Light curves of Mrk 501 with 30 day bins. The vertical bars represent the $1 \sigma$ uncertainties. The horizontal dashed lines and the legends (for all curves) show the results of a fit with a constant value to the data set.

period, the best fit with a constant value for the light curve is $(6.0 \pm 0.4) \times 10^{-4}$ counts $\mathrm{cm}^{-2} \mathrm{~s}^{-1}$ with a $\chi^{2} /$ ndf of $69.3 / 38$. This result could be evidence for small X-ray variability before this flaring period. Looking at a close-up view of the Swift/BAT light curve at $15-50 \mathrm{keV}$ shown in Figure 2, the large flare began on 2011 October 17 (MJD = 55851) and decreased to a low-activity state around the average level on November 22 (MJD = 55887; hereafter flare 1). Afterward, Mrk 501 became increasingly active for a longer period until about 2012 April. Its brightest flaring episode was on 2011 November 8, during the flare 1 period.

The Fermi-LAT data were analyzed using ScienceTools. ${ }^{25}$ The light curve was generated using aperture photometry. Panel (b) of Figure 1 shows the flux at energies greater than $0.3 \mathrm{GeV}$ that is contained within a $2^{\circ}$ cone centered on Mrk 501, which is the $68 \%$ containment angle of the reconstructed incoming photon direction for normal

\footnotetext{
25 http://fermi.gsfc.nasa.gov/ssc/
}

incidence. A fit with a constant value yields a $\chi^{2} /$ ndf of $107.7 / 44$ indicating a moderately variable behavior consistent with the X-ray analysis. The discrete correlation function (DCF), computed as prescribed by Edelson \& Krolik (1988), for the BAT/LAT data points shown in Figure 1 is DCF = $0.63 \pm 0.26$ for a time lag of zero, which is greater than the previously measured $\mathrm{DCF}=0.32 \pm 0.22$ (Abdo et al. 2011). Since the significance in both analyses is less than $2.5 \sigma$, only minor correlations are observed. During the X-ray flaring period, the $\mathrm{GeV} \gamma$-ray flux increased above the long-term average, but this flux increase is not significant. Therefore, the light curve does not indicate a significant correlation with the $\mathrm{X}$-ray data during the flare.

The light curve in the $\mathrm{TeV} \gamma$-ray range detected by ARGO-YBJ is shown in panel (c) of Figure 1. During the X-ray flare, the flux of $\mathrm{TeV} \gamma$-rays also increases. A fit with a constant emitting rate yields a $\chi^{2} /$ ndf of $71.9 / 44$, while the $\chi^{2} / \mathrm{ndf}$ is reduced to $42.59 / 38$ simply by excluding the data during the $\mathrm{X}$-ray flares. The $\mathrm{TeV} \gamma$-ray flare 1 was clearly detected as a 


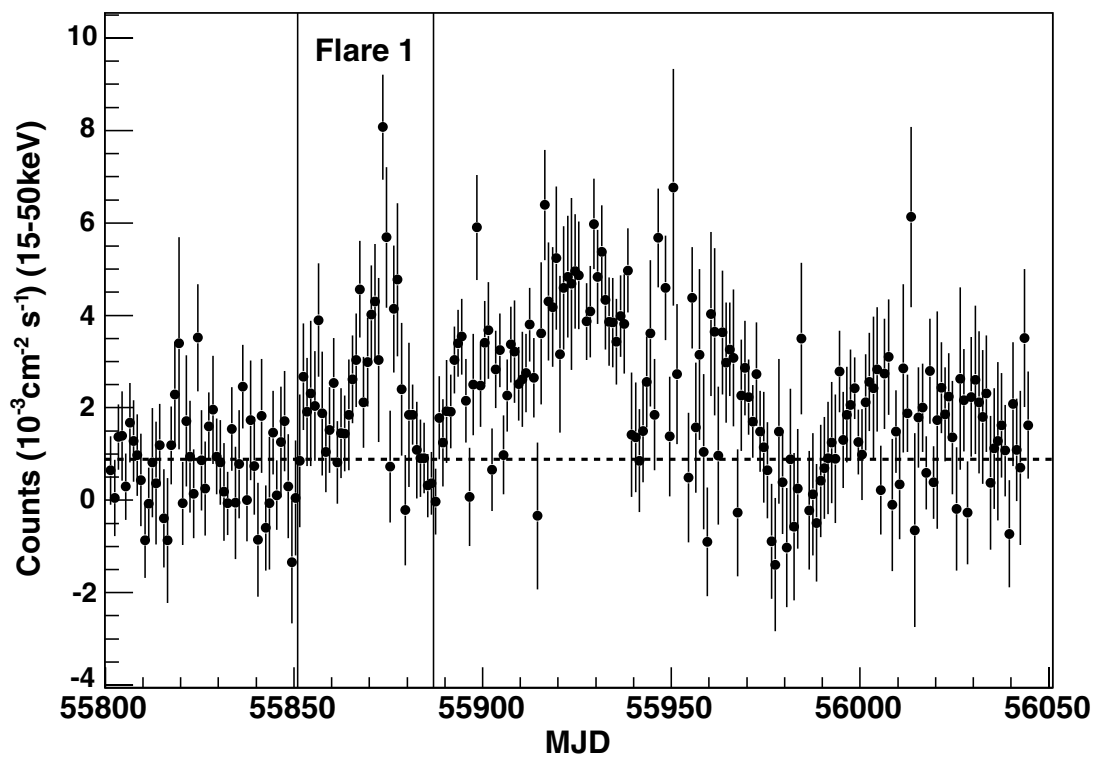

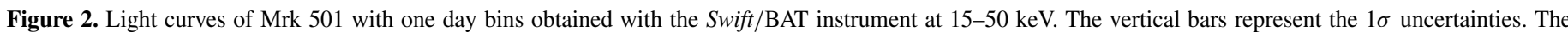
vertical lines show the start and the end of flare 1 . The horizontal dashed line is the same as that in Figure 1.
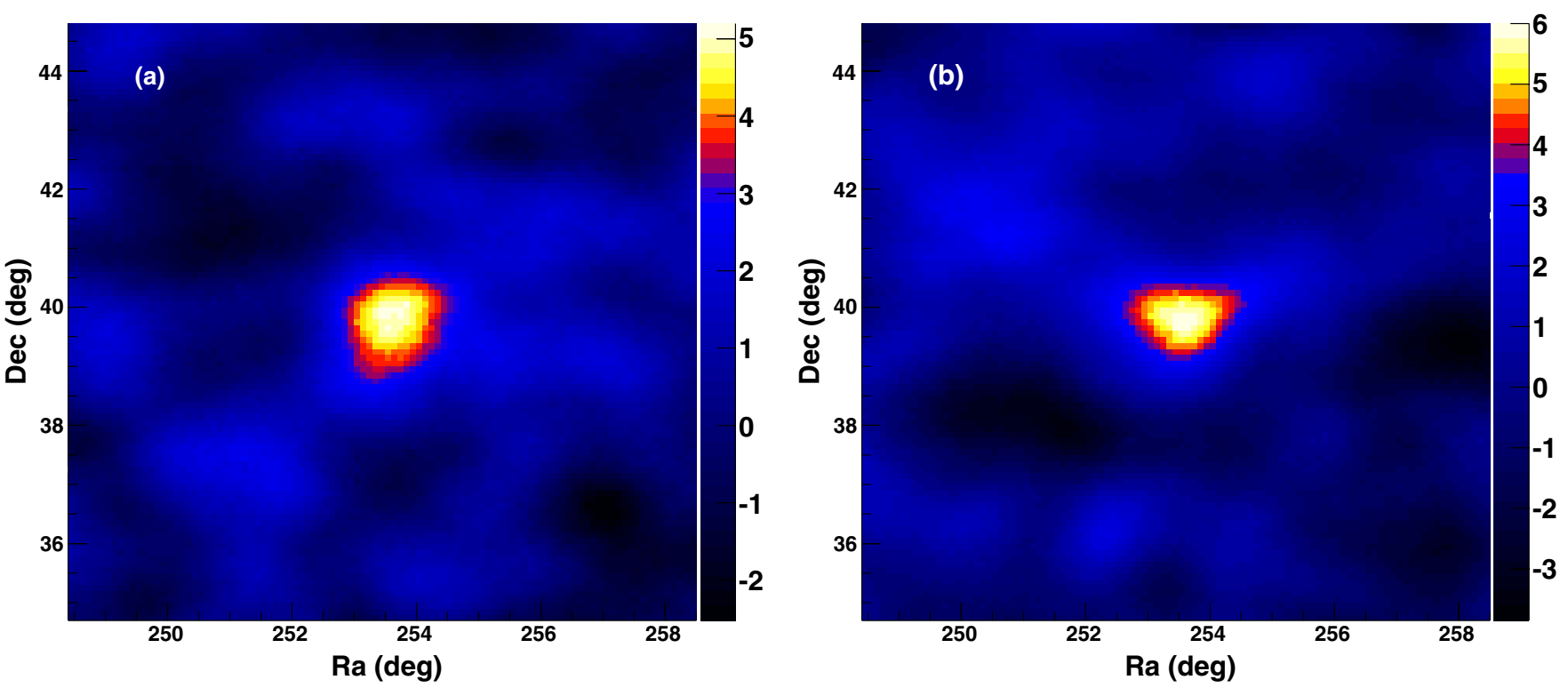

Figure 3. Significance map of the Mrk 501 region: panel (a) shows the statistical significance in standard deviations in the period from MJD 54683 to 55850; panel (b) shows the statistical significance of the flaring period from MJD 55851 to 55887.

(A color version of this figure is available in the online journal.)

counterpart of that in the X-ray band, and the count rate increase is by a factor of about six. The DCF for the BAT/ARGO-YBJ data points shown in Figure 1 is $0.85 \pm 0.36$ for a time lag of zero, while the DCF for the LAT/ARGO-YBJ is $0.44 \pm 0.31$.

\subsection{Photon Energy Spectra}

To investigate the evolution of the spectra, their time averages during the long-term quasi-steady state from 2008 August 5 to 2011 October 16 and during flare 1 are estimated separately. The integrated flux from Mrk 501 observed by ARGO-YBJ has a statistical significance of $5 \sigma$ (see panel (a) of Figure 3). During flare 1, the flux from Mrk 501 was detected by ARGO-YBJ with a $6.1 \sigma$ significance (see panel (b) of Figure 3), corresponding to an increase of the $\gamma$-ray flux above $1 \mathrm{TeV}$ by a factor of 6.6 from its steady emission. Events with $N_{\text {pad }}>60$ are used for both panels of Figure 3. For comparison, all of the spectra are shown in Figure 6, where an average spectrum over 4.5 months was adapted from Abdo et al. (2011). Since no significant activity was observed in the three years prior to flare 1, the average spectra over 4.5 months could approximately represent the average spectra over three years. All the other spectra presented in Figure 6 are estimated as follows.

\subsubsection{Swift: X-Ray}

The Swift/XRT is a focusing X-ray telescope with a energy range from 0.2 to $10 \mathrm{keV}$. In Windowed Timing mode during flare 1, XRT data in four time windows are available from HEASARC, ${ }^{26}$ and the exposure time is about $1 \mathrm{ks}$

\footnotetext{
${ }^{26} \mathrm{http}: / /$ heasarc.gsfc.nasa.gov/
} 
in each window. The XRT data set was processed with the XRTDAS software package (v.2.6.0) following the standard recommendations. The XRT average spectrum in the $0.5-10 \mathrm{keV}$ energy band was fitted using the XSPEC package (v.12.7.0). We adopted a power-law model for the photon-flux spectral density, with an absorption hydrogen-equivalent column density fixed to the Galactic value in the direction of the source, namely $1.56 \times 10^{20} \mathrm{~cm}^{-2}$ (Kalberla et al. 2005). We obtain the spectrum of $(0.0407 \pm 0.0007) \times(E / 1 \mathrm{keV})^{-1.824 \pm 0.022}$ photon $\mathrm{cm}^{-2} \mathrm{~s}^{-1} \mathrm{keV}^{-1}$.

\subsubsection{Fermi-LAT: HE $\gamma$-Rays}

The LAT data from a region centered on Mrk 501 with a radius of $10^{\circ}$ were used to estimate the spectrum. The data analysis was performed following the standard recommendations by using an unbinned maximum-likelihood method to estimate the source SED. We adopted a powerlaw model for the energy range from 0.1 to $300 \mathrm{GeV}$. During the long-term period, Mrk 501 was detected with a test statistic $(\mathrm{TS})$ value of $5118.2(\sim 71.5 \sigma)$. We obtain the spectrum $(2.300 \pm 0.068) \times 10^{-12}(E / 1844.3 \mathrm{MeV})^{-1.774 \pm 0.021}$ photon $\mathrm{cm}^{-2} \mathrm{~s}^{-1} \mathrm{MeV}^{-1}$. This is consistent with the result reported by the LAT collaboration using the data collected during the first 24 months (Abdo et al. 2012). The spectrum was found to be very stable in the $\mathrm{GeV}$ band despite a moderate variability over a long period. During flare 1 , the TS was $329.1(\sim 18.1 \sigma)$ and the spectrum was $(3.53 \pm 0.46) \times 10^{-12}(E / 1844.3 \mathrm{MeV})^{-1.640 \pm 0.084}$ photon $\mathrm{cm}^{-2} \mathrm{~s}^{-1} \mathrm{MeV}^{-1}$. Compared with the long-term result, the flux increased slightly.

\subsubsection{ARGO-YBJ: VHE $\gamma$-Rays}

The VHE $\gamma$-ray spectrum was estimated using a distribution of the excess in the number of events as a function of $N_{\text {pad }}$. We follow a widely used procedure, which is described in Bartoli et al. (2011a). In this procedure, the spectrum of Mrk 501 is assumed to be a power law. The ARGO-YBJ detector response has been taken into account using G4argo (Guo et al. 2010). The simulated events are sampled in the energy range from $10 \mathrm{GeV}$ to $100 \mathrm{TeV}$. We define six intervals with $N_{\text {pad }}$ of 20-59, 60-99, 100-199, 200-499, 500-999, and $\geqslant 1000$. The best fit gives a differential flux $\left(\mathrm{cm}^{-2} \mathrm{~s}^{-1} \mathrm{TeV}^{-1}\right)$

$$
(1.92 \pm 0.44) \times 10^{-12}\left(\frac{E}{2 \mathrm{TeV}}\right)^{-2.59 \pm 0.27}
$$

for the long-term period, corresponding to $0.312 \pm 0.076 \mathrm{Crab}$ above $1 \mathrm{TeV}$. The median energies of the six intervals are 0.45 , $0.89,1.4,2.8,5.6$, and $11 \mathrm{TeV}$, respectively. Both the flux and the spectral index are similar to those obtained by VERITAS and MAGIC during the low-activity state, as presented in Abdo et al. (2011). In particular, the ARGO-YBJ data show a smooth extension of the Fermi-LAT results, as evident in Figure 6. During flare 1 , the differential flux $\left(\mathrm{cm}^{-2} \mathrm{~s}^{-1} \mathrm{TeV}^{-1}\right)$ is

$$
(2.92 \pm 0.52) \times 10^{-12}\left(\frac{E}{4 \mathrm{TeV}}\right)^{-2.07 \pm 0.21}
$$

corresponding to $2.05 \pm 0.48 \mathrm{Crab}$ above $1 \mathrm{TeV}$, which is a factor of $6.6 \pm 2.2$ compared with its long-term steady state. The median energies of the six intervals are $0.89,1.1,1.8,3.5$, 7.1 , and $14 \mathrm{TeV}$, respectively. Only the statistical error is quoted here, and the systematic uncertainty in the flux measurement is estimated to be $\lesssim 30 \%$ (Aielli et al. 2010).

\section{DISCUSSION}

\subsection{Spectra Corrected for Extragalactic Background Light Absorption}

During flare $1, \gamma$-rays with a median energy of $8.4 \mathrm{TeV}$ are also observed with a significance greater than $4 \sigma$, as shown in Figure 4. $\gamma$-rays with such a high energy from Mrk 501 have not been detected since the 1997 flare. The SED at energies above $0.9 \mathrm{TeV}$ is harder than those observed during the flares in 1997 (Aharonian et al. 2001) and in the 2005 June 30 flare (Albert et al. 2007), as shown in Figure 6, although the spectral indices are consistent if the statistical error is taken into account. Mrk 501 is a nearby source, so we do not expect a significant absorption of its intrinsic source spectrum due to extragalactic background light (EBL) at energies below $1 \mathrm{TeV}$, while the absorption at higher energies is still considerable. Therefore, it is useful to test different EBL models assuming a minimum intrinsic photon spectral index. A natural minimum spectral index is 1.64 , constrained by the spectrum in the GeV band, since that should be steeper at higher energies.

Here we use four kinds of models with different flux levels of the EBL, among many models. Assuming a single power law for the VHE flux, the indices of the derived unabsorbed spectra using different models are (1) $1.80_{-0.29}^{+0.26}$ for the "low-IR" model proposed by Kneiske et al. (2004), which gives a result similar to that obtained using the EBL model of Aharonian et al. (2006), according to Albert et al. (2007), (2) $1.45_{-0.42}^{+0.36}$ for the model of Franceschini et al. (2008), which is widely used to correct the VHE SED of extragalactic sources, (3) and (4) $1.30_{-0.38}^{+0.34}$ and $1.11_{-0.41}^{+0.37}$ for the baseline and fast evolution models proposed by Stecker et al. (2006, 2007), respectively. For comparison, all of the unabsorbed spectra are shown in Figure 5. The spectral indices obtained using models (2)-(4) exceed the minimum spectral index boundary of 1.64; however, they are consistent with this limit if the statistical error is taken into account. Note that models (3) and (4) have been excluded with higher significance by previous tests carried out around $1 \mathrm{TeV}$ (Aharonian et al. 2006; Georganopoulos et al. 2010) and tens of GeV (Abdo et al. 2009, 2010). Since our data extend to about $10 \mathrm{TeV}$, the corresponding EBL photon energy is substantially lower. The EBL model with minimum absorption is used when modeling the SED in the following section.

\subsection{Modeling of the Overall SED}

The long-term averaged SED, especially the continuous measurement of the second component in the energy range $0.1 \mathrm{GeV}-10 \mathrm{TeV}$ obtained in this work, provides a robust baseline for insight into the underlying physics of Mrk 501. Over the very wide radio-VHE energy range, we fitted a one-zone SSC model proposed by Mastichiadis \& Kirk (1995; see also Mastichiadis \& Kirk 1997; Yang et al. 2008) to the SED. There are a few free parameters to be determined in this model, including the Doppler factor $\delta=1 /[\Gamma(1-\beta \cos \theta)]$, the spherical blob radius $R$, the magnetic field strength $B$, the electron spectral index $s$, the electron maximum Lorentz factor $\gamma_{\max }$, and the electron injection compactness $l_{e}=1 / 3 m_{e} c \sigma_{\mathrm{T}} R^{2} \int_{1}^{\infty} d \gamma(\gamma-1) Q_{e}$. In order to determine the Doppler factor and the injection compactness, further parameters must be determined, i.e., the Lorentz factor $\Gamma$, the speed of the blob $c \beta$, and the Lorentz factor of electrons $\gamma$. Moreover, $\sigma_{\mathrm{T}}$ is the Thomson cross section, $\theta$ is the angle between the direction of motion of the blob and the observer's line of sight, and $Q_{e}$ is the electron spectrum at injection, which is assumed to be a power-law cutoff at $\gamma_{\max }$, with a 


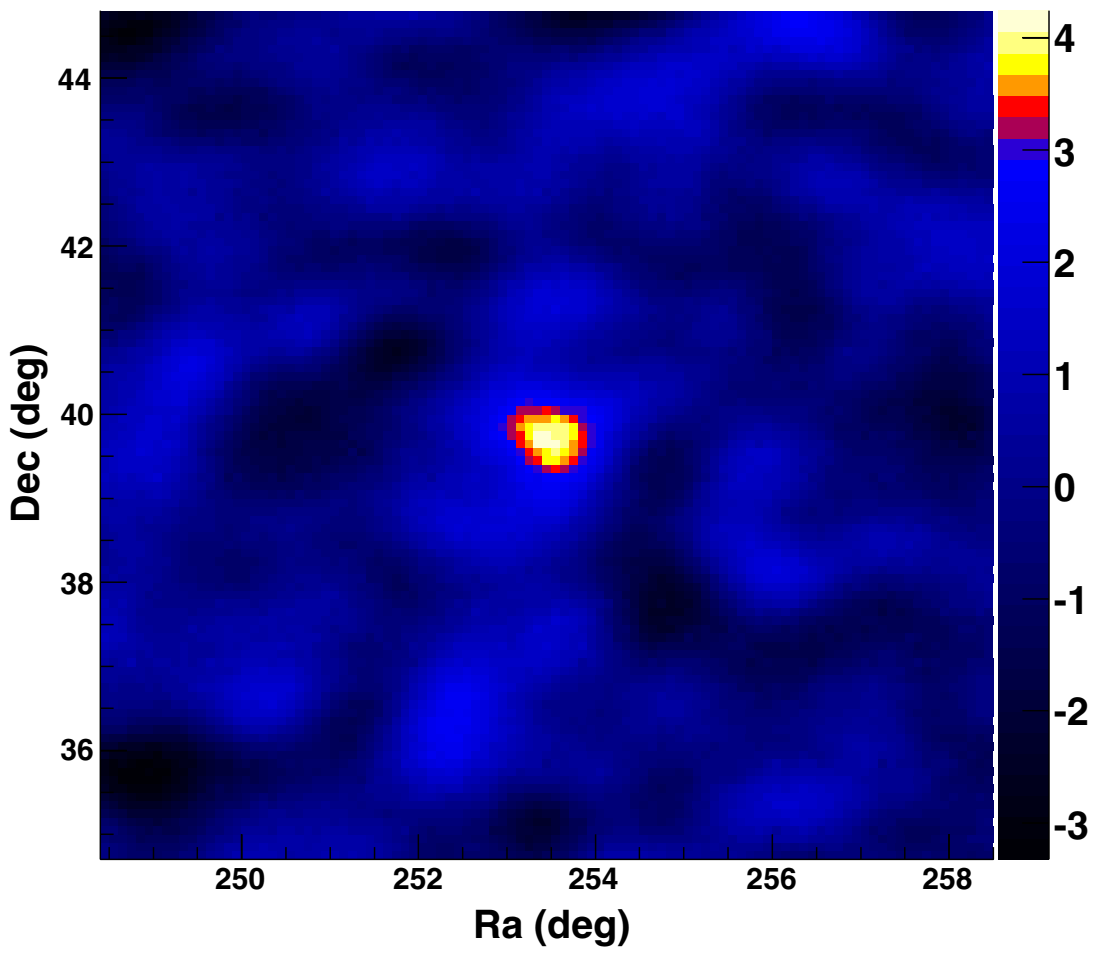

Figure 4. Significance map of the Mrk 501 region during the flaring period from MJD 55851 to 55887 . Events with $N_{\text {pad }}>500$ are used, whose corresponding median energy is $8.4 \mathrm{TeV}$.

(A color version of this figure is available in the online journal.)

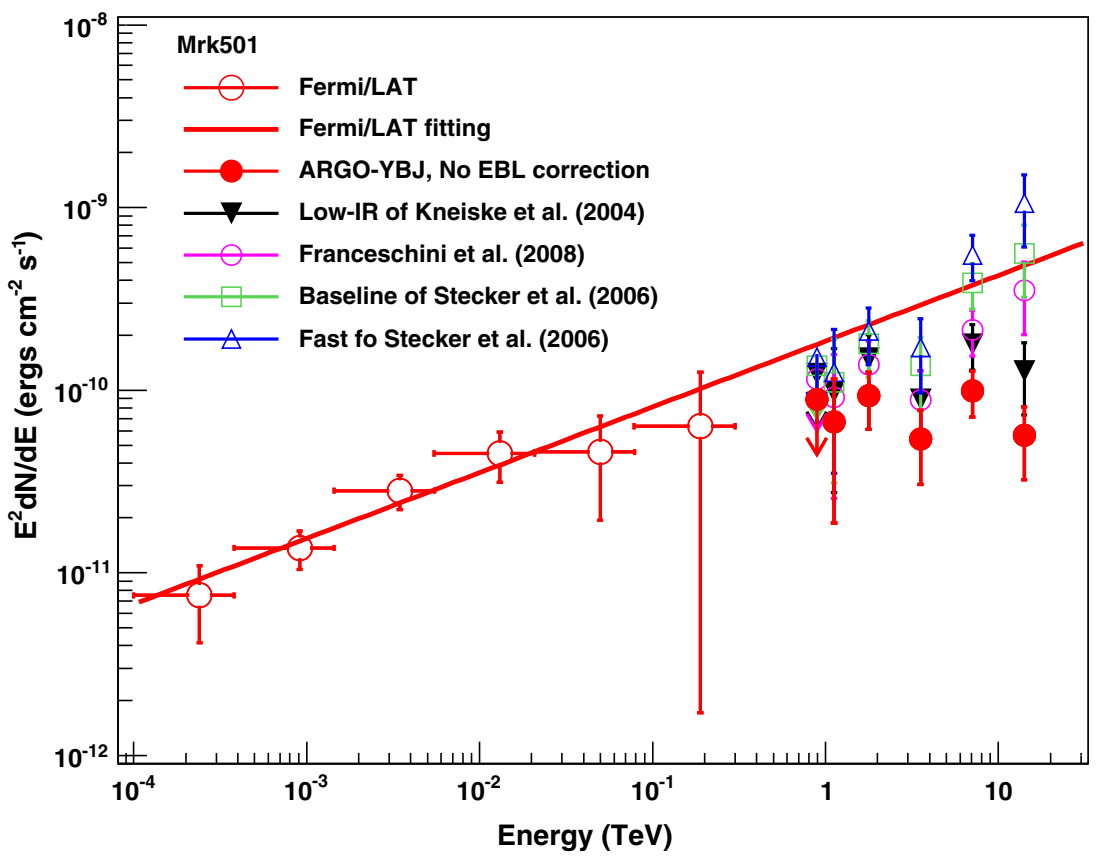

Figure 5. Spectra of Mrk 501 during the flaring period from MJD 55851 to 55887 . The spectrum from $0.1 \mathrm{GeV}$ to $300 \mathrm{GeV}$ is observed by Fermi-LAT. The solid line is the fit to the Fermi-LAT data using a power law. The spectrum observed by ARGO-YBJ has been corrected for EBL absorption using four different EBL models. Details can be found in the text.

(A color version of this figure is available in the online journal.)

normalization factor $q_{e}, Q_{e}=q_{e} \gamma^{-s} \exp \left(-\gamma / \gamma_{\max }\right)$. This model is different from other one-zone SSC models which introduce more free parameters using stationary injected electron spectra with a double power-law function (Tavecchio et al. 2001; Anderhub et al. 2009) or even triple power-law function (Abdo et al. 2011) with an exponential cutoff at the high-energy end. A full time-dependent evolution of the electron and photon spectra based on this model was simulated for a given injection spectrum of electrons. The low-IR model proposed by Kneiske et al. (2004) is used to take into account the absorption of $\gamma$-rays in the EBL when modeling the SED.

The best fit to the long-term SED is shown in Figure 6, with the corresponding parameters given in Table 1. General agreement between the model and the data is achieved with this 


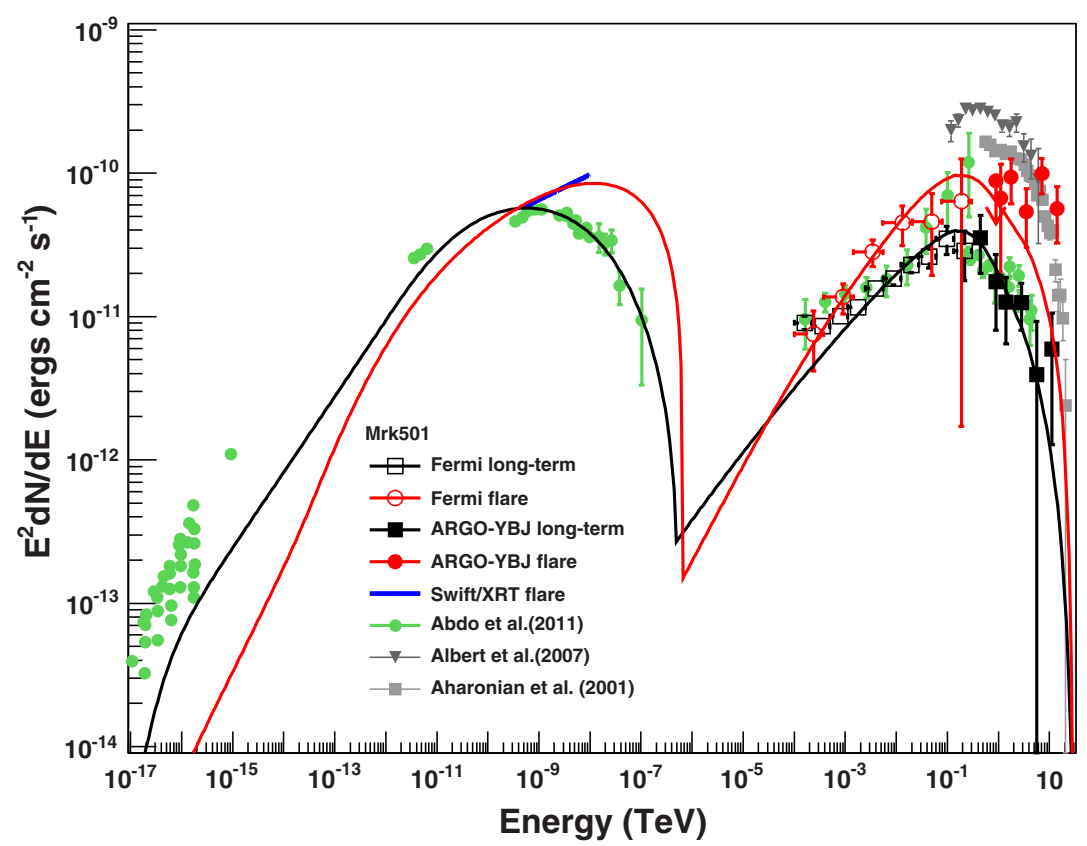

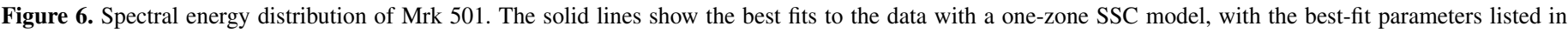
Table 1. The black curve corresponds to an SED model describing the long-term averaged data, while the red curve describes the flaring data.

(A color version of this figure is available in the online journal.)

Table 1

Best-fit Parameters in the SSC Model

\begin{tabular}{lcrcrcc}
\hline \hline Flux Level & $\gamma_{\max }$ & $l_{e}$ & $\begin{array}{c}B \\
(\mathrm{G})\end{array}$ & $\begin{array}{c}R \\
(\mathrm{~cm})\end{array}$ & & $s$ \\
\hline Long-term & $3 \times 10^{6}$ & $1.8 \times 10^{-5}$ & 0.07 & $3 \times 10^{16}$ & 12 & 1.95 \\
Flare 1 & $8 \times 10^{6}$ & $8 \times 10^{-5}$ & 0.10 & $1 \times 10^{16}$ & 10 & 1.6 \\
\hline
\end{tabular}

simple one-zone SSC model. The parameters in the model are found in general agreement with those found in the previous analysis on the similar source Mrk 421 (Bartoli et al. 2011a). The best fit for flare 1 is also shown in Figure 6, with the corresponding parameters given in Table 1. The highest-energy data points of the SED $(>6 \mathrm{TeV})$ during flare 1 cannot be well reproduced by only modifying the parameters. It is important to point out that the X-ray spectrum becomes harder during the flare. The peak energy is shifted to about $10 \mathrm{keV}$ during flare 1 from about $1 \mathrm{keV}$ in the quasi-steady state. The shift of the $\mathrm{X} / \gamma$-ray peak to higher energies during flares is a common feature that has been reported many times (e.g., Albert et al. 2007; Anderhub et al. 2009; Acciari et al. 2011). However, the detection of $>6 \mathrm{TeV} \gamma$-rays with an energy flux similar to that at $1 \mathrm{TeV}$ is unusual. In the framework of the SSC model, it is difficult to reproduce such high-energy $\gamma$-rays as detected by ARGO-YBJ. Since the $\gamma$-rays above $1 \mathrm{TeV}$ are typically produced in the Klein-Nishina regime, their rate should be strongly suppressed. The radiation mechanism during flares may be different from that in the quasi-steady state. Different radiation mechanisms, such as more complex SSC models or hadronic processes (e.g., in Abramowski et al. 2012), are needed to improve the understanding of the flaring phenomena.

\section{SUMMARY}

We have presented continuous long-term monitoring of Mrk 501 from 2008 August to 2012 April. Both the flux and the spectral index are consistent with those obtained by VERITAS and MAGIC during a 4.5 month multi-frequency campaign (Abdo et al. 2011). Combining the observations by the ARGO-YBJ experiment with the space-borne experiments with Swift and Fermi, the investigation was performed over a wide energy range from $0.5 \mathrm{keV}$ up to $10 \mathrm{TeV}$ (a value higher than the maximum in Abdo et al. 2011). Using all the data covering various energy bands during the quasi-steady phase of the blazar, its SED is fitted with a simple one-zone SSC model assuming a single power law with exponential cutoff for the electron spectrum at injection. The model parameters are found to be in agreement with those resulting from previous analyses for various AGNs, indicating that similar radiation mechanisms are in action. A strong flare of the blazar in the VHE region was observed by ARGO-YBJ in 2011 October, while no Cherenkov Telescope observations of Mrk 501 were possible during this period. It is well determined to be a counterpart of the X-ray flare in the same time period, giving for the BAT/ARGO-YBJ data points a DCF of $0.85 \pm 0.36$ for a time lag of zero. On the contrary, there is no significant increase of the flux at energies around $1 \mathrm{GeV}$. Remarkably, $\gamma$-rays with energies above $8 \mathrm{TeV}$ are detected, which has not happened since the 1997 flare. The spectral shape obtained with the data in the GeV/TeV energy region during the flare favors the "low-IR" EBL model of Kneiske et al. (2004), while a simple one-zone SSC model with a single power-law electron spectrum at injection is not able to reproduce the spectral shape at the highest energies (above $6 \mathrm{TeV})$.

This work is supported in China by the NSFC (grant No. 10120130794), the Chinese Ministry of Science and Technology, the Chinese Academy of Sciences, the Key Laboratory of Particle Astrophysics, CAS, and in Italy by the Istituto Nazionale di Fisica Nucleare (INFN).

We are grateful to Yupeng Chen for his help in estimating the $\mathrm{X}$-ray spectrum using the $S$ wift/XRT data. We also acknowledge essential support from W. Y. Chen, G. Yang, X. F. Yuan, C. Y. 
Zhao, R. Assiro, B. Biondo, S. Bricola, F. Budano, A. Corvaglia, B. D’Aquino, R. Esposito, A. Innocente, A. Mangano, E. Pastori, C. Pinto, E. Reali, F. Taurino, and A. Zerbini in the installation, debugging, and maintenance of the detector.

\section{REFERENCES}

Abdo, A. A., Ackermann, M., Ajello, M., et al. 2009, ApJ, 706, L138 Abdo, A. A., Ackermann, M., Ajello, M., et al. 2010, ApJ, 723, 1082

Abdo, A. A., Ackermann, M., Ajello, M., et al. 2011, ApJ, 727, 129

Abdo, A. A., Ackermann, M., Ajello, M., et al. 2012, ApJS, 199, 31

Abramowski, A., Acero, F., Aharonian, F., et al. 2012, A\&A, 539, A149

Acciari, V. A., Arlen, T., Aune, T., et al. 2011, ApJ, 729, 2

Aharonian, F. A. 2000, New Astron., 5, 377

Aharonian, F. A., Akhperjanian, A. G., Barrio, J. A., et al. 1999, A\&A, 342, 69

Aharonian, F. A., Akhperjanian, A. G., Barrio, J. A., et al. 2001, A\&A, 366, 62

Aharonian, F. A., Akhperjanian, A. G., Bazer-Bachi, A. R., et al. 2006, Nature, 440, 1018

Aielli, G., Assiro, R., Bacci, C., et al. 2006, Nucl. Instrum. Methods Phys. Res. A., 562, 92

Aielli, G., Bacci, C., Bartoli, B., et al. 2009a, Astropart. Phys., 30, 287

Aielli, G., Bacci, C., Bartoli, B., et al. 2009b, Astropart. Phys., 32, 47

Aielli, G., Bacci, C., Bartoli, B., et al. 2009c, Nucl. Instrum. Methods Phys. Res. A, 608, 246

Aielli, G., Bacci, C., Bartoli, B., et al. 2010, ApJ, 714, L208

Albert, J., Aliu, E., Anderhub, H., et al. 2007, ApJ, 669, 862

Amenomori, M., Ayabe, S., Cao, P. Y., et al. 2000, ApJ, 532, 302

Anderhub, H., Antonelli, L. A., Antoranz, P., et al. 2009, ApJ, 705, 1624
Bartoli, B., Bernardini, P., Bi, X. J., et al. 2011a, ApJ, 734, 110

Bartoli, B., Bernardini, P., Bi, X. J., et al. 2011b, Phys. Rev. D, 84, 022003

Bartoli, B., Bernardini, P., Bi, X. J., et al. 2011c, ATel, 3801

Bartoli, B., Bernardini, P., Bi, X. J., et al. 2012, ApJ, 745, L22

Cao, Z., \& Chen, S. Z. 2011, Proc. 32nd ICRC, Vol. 7, 211 (http://www.ihep.ac. cn/english/conference/icrc2011/paper/)

Dermer, C. D., Schlickeiser, R., \& Mastichiadis, A. 1992, A\&A, 256, L27

Djannati-Atai, A., Piron, F., Barrau, A., et al. 1999, A\&A, 350, 17

Edelson, R. R., \& Krolik, J. H. 1988, ApJ, 333, 646

Franceschini, A., Rodighiero, G., \& Vaccari, M. 2008, A\&A, 487, 837

Georganopoulos, M., Finke, J. D., \& Reyes, L. C. 2010, ApJ, 714, L157

Ghisellini, G., Celotti, A., Fossati, G., Maraschi, L., \& Comastri, A. 1998, MNRAS, 301, 451

Guo, Y. Q., Zhang, X.-Y., Zhang, J.-L., et al. 2010, Chin. Phys. C (HEP \& NP), 34,555

Kalberla, P. M. W., Burton, W. B., Hartmann, D., et al. 2005, A\&A, 440, 775

Kneiske, T. M., Bretz, T., Mannheim, K., \& Hartmann, D. H. 2004, A\&A, 413, 807

Krawczynski, H. 2004, New Astron. Rev., 48, 367

Li, T. P., \& Ma, Y. Q. 1983, ApJ, 272, 317

Mastichiadis, A., \& Kirk, J. G. 1995, A\&A, 295, 613

Mastichiadis, A., \& Kirk, J. G. 1997, A\&A, 320, 19

Quinn, J., Akerlof, C. W., Biller, S., et al. 1996, ApJ, 456, L83

Sikora, M., Begelman, M. C., \& Rees, M. J. 1994, ApJ, 421, 153

Sootome, T., Sugizaki, M., Mihara, T., et al. 2011, ATel, 3752

Stecker, F., Malkan, M. A., \& Scully, S. T. 2006, ApJ, 648, 774

Stecker, F., Malkan, M. A., \& Scully, S. T. 2007, ApJ, 658, 1392

Tavecchio, F., Maraschi, L., Pian, E., et al. 2001, ApJ, 554, 725

Yang, C. Y., Fang, J., Lin, G. F., \& Zhang, L. 2008, ApJ, 682, 767 